\title{
Clinical Vignettes Automatic Errors: A Case Series on the Errors Inherent in Electronic Prescribing
}

\author{
Laura M. Lourenco, PharmD, BCPS' , Adam Bursua, PharmD², and Vicki L. Groo, PharmD \\ 'Department of Pharmacy, University of Chicago, Chicago, IL, USA; ${ }^{2}$ Department of Pharmacy Practice (MC 886), University of Illinois at Chicago \\ College of Pharmacy, Chicago, IL, USA.
}

The adoption of electronic prescribing is on the rise, as it reduces medication errors compared to handwritten orders. The inadvertent dispensing of discontinued medications is a type of medication error that is less well described, but one that can lead to adverse events. Software for electronic prescriptions transmits orders for refills or new prescriptions, but not discontinuations, to the pharmacy. Medications that have been stopped are displayed only at the prescribing facility's electronic medical record (EMR). This report describes five cases in which the pharmacy dispensed electronically discontinued medications, two of which contributed to adverse outcomes.

KEY WORDS: medication error; electronic prescribing; transitions of care. J Gen Intern Med 31(7):808-11

DOI: $10.1007 / \mathrm{s} 11606-016-3606-5$

(C) Society of General Internal Medicine 2016

\section{BACKGROUND}

The Institute of Medicine defines medication errors as any error in the medication use process, which encompasses prescribing, transcribing, administering, and monitoring. ${ }^{1} \mathrm{Al}-$ though electronic prescribing (e-prescribing) has been shown to reduce medication errors and adverse events, most of the reported data have been in the inpatient setting. ${ }^{2,3}$ A study of community-based office practices found that prescribing errors were reduced from 42.4 to 6.6 per 100 prescriptions at 1 year among adopters of e-prescribing, while there was no change among non-adopters $(p<0.001){ }^{4}$

The inadvertent dispensing of discontinued medications is another form of medication error. Harvard Vanguard Medical Associates found that $1.5 \%$ of electronically discontinued medications were still being dispensed at the pharmacy during a 12-month follow-up, and $34 \%$ of these medications met criteria for higher risk of adverse events. ${ }^{5}$

E-prescribing is a component of meaningful use set forth by the Centers for Medicare \& Medicaid Service (CMS), and a steady annual increase in its adoption has been reported by Surescripts ${ }^{\circledR}{ }^{6}$ This technology is not without limitations, however, as it currently allows only for the transmission of new prescriptions or refills from the prescriber to the

Received April 27, 2015

Revised September 24, 2015

Accepted January 22, 2016

Published online February 16, 2016 pharmacy. Discontinuation orders are entered in the prescribing facility's EMR, but not transmitted to the pharmacy, thus resulting in discrepancies between provider and pharmacy medication lists, with potential for dispensing discontinued medications.

Here we report a series of cases in which e-prescribing contributed to medication errors. An exemption to informed consent requirements was granted from the University of Illinois Institutional Review Board.

\section{CASE 1}

A 37-year-old woman was referred to a cardiology clinic after being diagnosed with congestive heart failure during hospital admission. She was discharged on $10 \mathrm{mg}$ lisinopril, $25 \mathrm{mg}$ metoprolol succinate and $20 \mathrm{mg}$ furosemide daily. Over the next few outpatient visits, her metoprolol was increased to $75 \mathrm{mg}$ daily.

She was e-prescribed "metoprolol $50 \mathrm{mg} \times 1.5$ tabs." However, the pharmacy dispensed the previous prescription, "metoprolol $50 \mathrm{mg}$ daily, \#30." Consequently, she ran out of medication early, resulting in an insurance rejection when a refill was requested. The pharmacist was unable to determine the reason for the error, but suggested that the patient may have requested a refill using the old bottle. Despite confirmation that the prescription for the dose increase was received, the old prescription was still active and available for refill requests.

\section{CASE 2}

A 49-year-old man with congestive heart failure presented to the clinic with shortness of breath at rest, massive edema to the thighs, and a 10-kg weight gain. His pharmacy mistakenly filled a bumetanide prescription for $3 \mathrm{mg}$ twice daily (utilizing 1-mg tablets) 4 days after a new prescription was e-prescribed for $4 \mathrm{mg}$ twice daily. Because the patient took the increased dose, the old prescription did not provide a sufficient supply for 30 days, resulting in an insurance rejection when the refill was requested. Consequently, the patient was hospitalized for heart failure exacerbation for 7 days, having exhausted his diuretic supply a week early. Similar to case 1, the pharmacy confirmed receipt of the new prescription for the dose increase 
and had two active prescriptions on file for the same medication, but could not explain why the higher dose had not been dispensed. Furthermore, the pharmacist confirmed that the patient had asked for his diuretic, but because it was a Friday afternoon, the insurance provider could not be reached for approval to dispense additional medication, and did the pharmacist did not offer the patient a temporary supply until the issue could be resolved.

\section{CASE 3}

Upon discharge of a 25-year-old woman hospitalized for lupus exacerbation, e-prescriptions were sent to her pharmacy. In addition to new prescriptions related to her hospitalization, several prescriptions for the patient's chronic medications were e-prescribed. Among these was hydroxychloroquine. Unfortunately, the prescriber mistakenly wrote an e-prescription for a look-alike/sound-alike drug, hydroxyurea. The prescriber caught the error just a few moments after signing the e-prescription, and the order for hydroxyurea was discontinued. However, that order did not prompt a "stop" or "discontinue" notice to the pharmacy. Approximately 1 month later, the patient was readmitted to the hospital with complaints of generalized weakness. Her pharmacy was contacted, and it was discovered that the patient had filled and been taking both the erroneous hydroxyurea prescription and the hydroxychloroquine. During the hospitalization, the patient was noted to have leukopenia (WBC $1100 / \mathrm{mm}^{3}$ ) and neutropenia (absolute neutrophil count $400 / \mathrm{mm}^{3}$ ), consistent with the toxicity profile of hydroxyurea. These hematological toxicities eventually resolved upon discontinuation of the drug.

\section{CASE 4}

A 58-year-old man with congestive heart failure and ventricular tachycardia presented to an electrophysiology clinic for routine monitoring of his amiodarone. Upon findings of fibrosis on chest $\mathrm{x}$-ray, the patient was instructed to discontinue his 200-mg amiodarone tablet. The medication was also cancelled in the EMR at the prescribing institution. At a follow-up visit 3 months later, it was found that the patient mistakenly discontinued his 200-mg metoprolol succinate tablets and continued the $200 \mathrm{mg}$ amiodarone. Similar to case 4, the discontinuation of a prescription in the local EMR did not transmit a stop order to the pharmacy, so the patient continued to receive refills of a medication that was causing an adverse event.

\section{CASE 5}

A 64-year-old woman with a drug-eluting stent was prescribed clopidogrel for 1 year. After 2 months, her prescription was changed to ticagrelor due to an adverse effect with clopidogrel.
The prescriber utilized the special instructions box in the eprescribing system, stating "Patient with rash on clopidogrel. This medicine replaces clopidogrel." Prescriptions were dispensed correctly until November, when the patient began receiving 30-day supplies of both clopidogrel and ticagrelor. The error was discovered in January, and the patient stated that she was not taking the clopidogrel. When asked why she was buying clopidogrel, she replied, "I call the store and ask them to send all of my medications." The pharmacy was contacted by phone and advised of the error, at which time the clopidogrel prescription was cancelled.

\section{DISCUSSION}

The emergence of novel technologies designed to improve health systems has allowed for more efficient patient care experiences and, in many ways, has facilitated more effective interdisciplinary communication. However, such advances in technology may be accompanied by a variety of limitations and untoward consequences. In each case described above, a change was made to a patient's medications. However, because of technology limitations and human error, each of these patients' pharmacotherapy regimes was deleteriously affected.

Cases 1 and 2 highlight issues with dose changes. In each scenario, the patient took an increased dose using their pill supply. When a refill was provided, the pharmacist did not utilize the most current prescription order, leading to the patient receiving an inadequate supply of medication. A specific root cause was not discovered, but we can postulate that one of the following occurred: 1) the patient unknowingly refilled the previous prescription, 2) the patient was enrolled in an automatic refill program that refilled the patient's previous dose, or 3) the pharmacist did not contact the prescriber regarding dose clarification.

Cases 3,4 , and 5 are a result of dispensing discontinued medications. In case 3 , the provider realized his mistake immediately but was unaware he needed to call the pharmacy to cancel the prescription. As a result, the patient received an unintended medication, resulting in serious adverse effects. In case 4 , although this patient did not experience an adverse outcome, amiodarone-induced pulmonary fibrosis is reported to be fatal in anywhere from 1 to $33 \%$ of cases. ${ }^{7,8}$ The patient's inadvertent discontinuation of the wrong medication exposed him to risk of worsening fibrosis, along with missing an important medication for his heart failure. In case 5, duplicate therapy was dispensed, subjecting the patient to the risk of bleeding. In each case, the medication was discontinued within the healthcare system's EMR. Since the stop orders for the hydroxyurea and amiodarone were not transmitted to the pharmacy, the pharmacists had no way of knowing not to fill them, nor do they have access to a patient's problem list. In case 5 , the provider took the extra step in stating that the new order for ticagrelor was replacing clopidogrel, but the pharmacist did not utilize or may not have seen this information. 
Finally, cases 2 and 3 illustrate the potential magnitude of system failure, as both resulted in hospitalization.

Each of these cases occurred within a single healthcare system using the same EMR (Cerner $\left.{ }^{\circledR}\right)$ for inpatient and outpatient encounters. The provider responsible for managing the disease was the prescriber of the medication change. The five cases occurred at four different pharmacies, each a community chain drug store.

To date, most research has focused on the evaluation of errors related to the implementation of e-prescribing software (data entry and electronic transmission). ${ }^{9-13}$ Cochran et al. found a $2.5 \%$ discrepancy rate between clinic eprescriptions and pharmacy labels. ${ }^{14}$ Nanji and colleagues characterized the unrealized potential and residual consequences of e-prescribing. ${ }^{15}$ Limitations in communication were described, highlighting the unidirectional (prescriber to pharmacy) flow of electronic information. Also noted was the fact that prescribers were unable to electronically cancel or modify prescriptions, and instead had to call the pharmacy to correct any errors. The frequency of errors described in this case series is unknown, but it highlights a systemic failure due to limitations in the e-prescribing software, human error in utilization of the system, and low patient literacy.

Improved methods of electronic communication between the clinic and the pharmacy are needed. The automatic transmission of stop orders to the pharmacy could prevent the dispensing of discontinued medications. The transmission of cancellation notices is contingent on both provider and pharmacy software functionality. Surescripts ${ }^{(B)}$ currently has 108 provider and 70 pharmacy software systems certified for eprescribing. ${ }^{16}$ The National Council for Prescription Drug Programs (NCPDP) includes cancellation of a prescription in the SCRIPT standard. ${ }^{17}$ The NCPDP SCRIPT implementation guide contains information for stakeholders to create software for the exchange of data. ${ }^{18}$ Until this type of transaction between EMR's and pharmacies is universal, the risk of these types of errors remains high.

With regard to dose changes, pharmacies do not automatically inactivate a drug when a new order for a different strength is received, as they do not immediately know the prescriber's intent. For example, a patient could have orders for 25 and $50 \mathrm{mg}$ hydralazine TID. The intent could be a dose increase or a patient using both orders to make up a $75-\mathrm{mg}$ TID dose. Software enhancements to allow the pharmacy to communicate electronically with the prescriber would enable pharmacists to obtain dose clarifications. ${ }^{15}$ Finally, including an indication on the prescription may help in the detection of medication errors such as the wrong patient or wrong drug, as described by Nanji et al. ${ }^{15}$ and shown in case 3 .

Until these enhancements can be made, healthcare systems should direct efforts toward increased awareness of eprescribing limitations and should educate prescribers accordingly. We recommend direct communication with pharmacies in cases where harm could result from the continued use of a medication. Patients should be provided with printed medication lists at each encounter and instructed to verify medications/doses with their pharmacy. The addition of notations to prescriptions to indicate the prescriber's intent may help improve communication to the pharmacist. Finally, pharmacists need to pay close attention to active prescriptions and reach out to the prescriber to clarify doses or duplications in therapy.

\section{CONCLUSION}

E-prescribing has been shown to reduce transcription errors, and utilization of this practice is on the rise. However, as evidenced by these cases, under-recognized limitations of the technology can lead to adverse outcomes. Software upgrades to enhance provider-pharmacy communication and to allow for discontinuation orders may help mitigate the risk of these types of medication errors. Until then, recognizing and educating providers on the limitations of e-prescribing is imperative.

Acknowledgments: No financial support was provided in relation to this work.

Corresponding Author: Vicki L. Groo, PharmD; Department of Pharmacy Practice (MC 886)University of Illinois at Chicago College of Pharmacy, 833 South Wood Street, 164 PHARM, Chicago, IL 606127230, USA (e-mail: vjust@uic.edu).

\section{Compliance with Ethical Standards:}

Conflict of Interest: Laura M. Lourenco: no conflicts to disclose Adam Bursua: stock options, currently has zero; Board of Advisors TG Healthcare Corp

Vicki L. Groo: speaker honoraria; Bristol Myers Squibb, Pfizer Inc.

\section{REFERENCES}

1. Kohn LT, Corrigan J, Donaldson MS, Institute of Medicine (US) Committee on Quality of Health Care in America. To err is human: building a safer health system. Washington: National Academy Press; 2000.

2. Ammenwerth E, Schnell-Inderst P, Machan C, Siebert U. The effect of electronic prescribing on medication errors and adverse drug events: a systematic review. J Am Med Inform Assoc. 2008;15:585-600.

3. Wolfstadt JI, Gurwitz JH, Field TS, et al. The effect of computerized physician order entry with clinical decision support on the rates of adverse drug events: a systematic review. J Gen Intern Med. 2008;23:451-58.

4. Kaushal R, Kern LM, Barrón Y, Guaresimo J, Abramson EL. Electronic prescribing improves medication safety in community-based office practices. J Gen Intern Med. 2010;25:530-36.

5. Allen SA, Sequist TD. Pharmacy dispensing of electronically discontinued medications. Ann Intern Med. 2012;157:700-05.

6. The National Progress Report on E-Prescribing and Safe-Rx Ranking, Year 2014. Available at: http://www.surescripts.com/news-center/nationalprogress-report-2014. Accessed December 15, 2015

7. Cordarone Product Monograph 2010. Available at: http://labeling.pfizer. com/showlabeling.aspx?id=93 Accessed December 15, 2015.

8. Dusman RE, Stanton MS, Miles MD, et al. Clinical features of amiodarone-induced pulmonary toxicity. Circulation. 1990;82:51-59.

9. Abramson EL, Barron Y, Quaresimo J, et al. Electronic prescribing within an electronic health record reduces ambulatory prescribing errors. Jt Comm J Qual Patient Saf. 2011;37:470-8.

10. Oren E, Shaffer ER, Guglielmo BJ. Impact of emerging technologies on medication errors and adverse drug events. Am J Health Syst Pharm. 2003;60:1447-58.

11. Eslami S, Abu-Hanna A, de Keizer NF. Evaluation of outpatient computerized physician medication order entry systems: a systematic review. J Am Med Inform Assoc. 2007;14:400-6. 
12. Nanju KC, Rothschild JM, Salzberg C, et al. Errors associated with outpatient computerized prescribing systems. J Am Med Inform Assoc. 2011;18:767-73.

13. Moniz TT, Seger AC, Keohan CA, et al. Addition of electronic prescription transmission to computerized prescriber order entry: effect on dispensing errors in community pharmacies. Am J Health Syst Pharm. 2011;68:15863

14. Cochran GL, Klepser DG, Morien M, et al. From physician intent to the pharmacy label: prevalence and description of discrepancies from a crosssectional evaluation of electronic prescriptions. BMJ Qual Saf. 2014;23:223-30.
15. Nanji KC, Rothschild JM, Boehne JJ, et al. Unrealized potential and residual consequences of electronic prescribing on pharmacy workflow in the outpatient pharmacy. J Am Med Inform Assoc. 2014;21:481-486.

16. Surescripts Network connections. Available at: http://surescripts.com/ network-connections. Accessed December 22, 2015.

17. NCPDP Electronic Prescribing Standards. Available at: http://www.ncpdp. org/Resources/ePrescribing (link NCPDP Resources - ePrescribing Basics) Accessed December, 22, 2015.

18. SCRIPT Implementation Recommendations. Available at: http://www. ncpdp.org/NCPDP/media/pdf/SCRIPT-Implementation-Recommendations. (V1.34 pages 131-133) Accessed December 22, 2015 